\title{
Title: Plasmodesmata: a signaling hub at the cellular boundary
}

Jung-Youn Lee*

Department of Plant and Soil Sciences, Delaware Biotechnology Institute, University of Delaware, Newark, Delaware 19711, USA

${ }^{*}$ To whom correspondence and requests for materials should be addressed.

E. mail: lee@dbi.udel.edu

Fax: (302) 831-3447 


\begin{abstract}
Effective intercellular communication is crucial for the survival of plants. Because plant cells are encased in rigid cell walls, direct cell-to-cell exchange of cytoplasmic content is only possible through plasmodesmata (PD), membrane-lined nanotubes that connect the cytoplasm of adjacent cells. PD are highly dynamic communication channels that can undergo various structural and functional modifications. Recent findings in the field suggest that defense signaling pathways are tightly linked to the regulation of PD, and the restriction of PD-mediated cell-to-cell communication is a critical innate immune response to microbial pathogens. Moreover, several plasma membrane-bound signaling components, including receptor-like kinases that are known to have non-cell autonomous function or pathogen perception at the cell periphery, are found to also partition to PD. These findings hint at the novel role of PD as a signaling hub for both symplasmic and cross-membrane pathways.
\end{abstract}

\title{
Introduction
}

Direct signaling across cellular boundaries of adjacent cells in plants mainly occurs via receptormediated apoplasmic and PD-mediated symplasmic pathways. PD are membrane-lined pores that bridge adjacent cells across the cell wall matrices, providing cytoplasmic and membrane continuities for direct cell-to-cell communication [1-3]. Because apoplasmic and symplasmic pathways have distinct characteristics in both molecular players and the signal trafficking mode, it has long been presumed that these two pathways mediate non-cell autonomous signaling in a non-overlapping manner. In the apoplasmic pathway, signal-generating cells secrete specific ligands into the extracellular matrix, and recipient cells perceive the ligands by producing membrane-anchored, cognate receptors on the cell surface. In the symplasmic pathway, signaling molecules are commonly thought to move cytoplasmically through the PD that connect signal-generating cells to their adjacent recipient cells [4]. The protein composition and the molecular architecture of PD are yet to be discovered, but an increasing number of proteins has been identified to localize or partially associate with PD [5-7]. Certain plasma membrane (PM)localized receptor-like kinases (RLKs) and membrane-anchored proteins are also found to associate with PD. Some participate in non-cell autonomous signaling via the apoplasmic pathway during cell differentiation, and others in immune responses [8-11]. Placing such receptors right at PD could serve as an effective mechanism for regulating molecular trafficking while processing specific apoplasmic signals.

The recruitment of specific PD-associated proteins to defense signaling as well as the dispatch of defense proteins to PD illustrate how the regulation of PD is integrated into specific cellular signaling pathways during biotic stress [3]. For example, basal immune responses require activation of various signaling pathways, including those mediated by defense hormones such as salicylic acid (SA) [12]. Elevated SA concentrations during pathogen infection induce a restriction in cell-to-cell movement via PD by transcriptionally upregulating the expression of the PD regulator PD-located protein 5 (PDLP5) [13]. Accumulation of PDLP5 at PD restricts PD permeability by stimulating PD callose deposition. Feedback amplifies SA levels, further augmenting the PD closure response [14]. Effective innate immune responses during interactions with microbial pathogens also involve the recognition of pathogens via membrane- 
bound receptors at the cell surface levels [15]. Certain immune receptors, including FLAGELLIN SENSING 2 (FLS2) and LYSIN MOTIF DOMAIN-CONTAINING

GLYCOSYLPHOSPHATIDYLINOSITOL-ANCHORED PROTEIN 2 (LYM2), are not only partitioned to $\mathrm{PD}$, but also required for restricting PD permeability upon pathogen infection [10]. Furthermore, when plant cells lose dynamic control over PD gating, both the basal defense and systemic acquired resistance are compromised $[10,13,16]$. Collectively, these data underscore the biological significance of the PD-mediated intercellular communication under biotic stress.

In the current review, I discuss recent findings with the goal of showing how PD may serve as a signaling hub in which receptor-mediated and other cellular signaling events are integrated. First, I describe the structural characteristics and subdomains of PD. I go on to examine how different PD-associated proteins might be targeted or partitioned to specific subdomains of PD. Lastly, I discuss how PD are regulated by different immure signaling pathways and speculate on what role the regulation of PD might play in coping with biotic stress.

\section{PD form distinct subdomains to which PD-proteins differentially partition}

Structurally, each plasmodesma contains a cytoplasmic sleeve formed between outer and inner membrane linings, which are continuous with the PM and the endoplasmic reticulum (ER), respectively [17] (Fig. 1). The sleeve is thought to provide a passageway for soluble molecules to move through. On the other hand, the two membrane linings may provide specialized lipophilic surfaces within which hypothetic proteinaceous materials are embedded, or to which cytoskeletal elements are attached [18]. Current understanding about the outer and inner PD membranes based on the lipid composition and trafficking studies holds that they likely differ in their physical and chemical properties from the PM and cortical ER, respectively [18]. In this review, I adopt the terms PDom for $\underline{P D}$ outer membrane and PDim for $\underline{P D} \underline{i n n e r}$ membrane linings to distinguish these from the PM and ER. I propose the use of PDim or appressed ER over desmotubule, as the latter is a historical misnomer and does not reflect the fact that this inner structure is derived from the ER [19-21].

PD pores can be further divided into distinct spatial subdomains: orifices (two ends of the cytoplasmic sleeves open to the cytoplasm), neck regions (PDom at the orifices), and the central cavity/region (plasmodesmal bulge occurring at the middle lamella). Callose, a type of $\beta$ 1-3 glucan polymer, is usually found surrounding the neck regions of PD in the extracellular space between the PM and the cell wall [22]. The levels of callose accumulation at PD fluctuate in response to many environmental and developmental factors, and negatively correlate with PD permeability $[3,23,24]$. An ultrastructural resolution using immunogold labeling combined with a statistical analysis is necessary to fine-map a subdomain with which a PD protein is specifically associated. Although this is technically demanding due to the nano-scale dimensions of PD, several PD-associated proteins, including viral and innate proteins, have been mapped to a certain subdomain or structure. For example, the movement protein encoded by the Tobacco mosaic virus (TMV MP) and the PDLP5 expressed in Arabidopsis thaliana accumulate at the PD central cavity region $[13,25,26]$. PDLP1, another member of the PD-located protein family, and CRINKLY 4, a maize receptor-like kinase (LRK), are distributed along the PDom $[27,28]$. PDLP1 negatively regulates PD permeability by an unknown mechanism [29]. Whether there are any effects of CRINKLY4 on PD function is not yet known. Members of PD-callose binding proteins (PDCBs) are located to the outer surface of the PM, but preferentially accumulate at the PD 
neck regions [30]. Certain members of the $\beta$ 1-3 glucanases (BGs) that associate with PD, such as PdBGs, are thought to localize to the PD neck regions similar to the PDCBs [31].

Overexpression of PdBG2 and PDCB1 facilitates an increase in PD permeability via callose degradation and restricts PD permeability through heightened PD callose accumulation, respectively [30,31]. Both the molecular basis for preferential targeting to specific PD subdomains and its differential impact on PD functionality of PD-associated proteins await elucidation. However, the fact that different proteins cluster at specific areas within PD implies that these nano-domains represent functionally, and perhaps structurally, distinct sections of PD.

It is notable that some proteins that target the same subdomain may not necessarily colocalize within the same PD. When TMV MP and PDLP5 are coexpressed as fluorescently-tagged proteins, their signals do not completely overlap, seemingly excluding each other from occupying the same PD [13]. The proteins' mutual exclusion or competition for the same PD subdomain may be attributed to their opposing functions. For example, both TMV MP and PDLP5 function in altering PD permeability, but in an opposite manner: TMV MP facilitates an increase in PD permeability, and PDLP5 facilitates a decrease. PDLP5 induces a PD closure by stimulating PD callose deposition [14], and overexpression of PDLP5 interrupts cell-to-cell trafficking of the MP alone or the whole TMV [13]. It is not known whether TMV MP exerts PD gating by modulating PD callose levels. However, given that TMV movement is subjected to altered PD callose levels determined by endogenous genetic factors [13,32], it is unlikely that TMV MP induces PD opening by manipulating callose-dependent PD gating mechanism.

\section{Sterol-rich PD subdomain may attract certain GPI-linked extracellular proteins}

A recent PD lipidomics study showed that the primary PD isolated from $A$. thaliana suspension cultured cells were enriched with PDom, and that this membrane fraction contained relatively higher levels of sterols and complex sphingolipids than the PM [33]. This study also demonstrated that altering sterol content blocks PD partitioning of two glycosylphosphatidylinositol (GPI)-anchored PD-associated proteins: PDCB1 and PdBG2. GPI attachment is a post-translational modification that tethers secretory proteins to the extracellular periphery of the PM. Transgenic Arabidopsis seedling roots treated with sterol inhibitors decreased PD-association and increased PM distribution of PDCB1-GFP and PdBG2-GFP. Given that PDCB1 partitions to the neck region of PD, it seems reasonable to interpret this finding as evidence that sterol-rich lipids might be more concentrated within the neck region of PDom.

The drug treatment had no impact on the localization pattern of the type I transmembrane (TM) protein PDLP1, which distributes along the PDom [28] and has PD targeting dependentent on the TM helix [29]. This observation raises the interesting question of whether or not the sterolrich PD neck regions form some sort of a membrane border that separates PDom from the PM. This separation could help prevent lateral diffusion of PM proteins into PDom, while permitting a localized accumulation of GPI-anchored PD proteins. Along these lines, it is noteworthy that although PDLP family members are characterized by a single TM helix flanked by two Cys-rich ectodomains and a short cytoplasmic tail, they slightly differ in their localization patterns: PDLP5 localizes to the central region of PD and does not have PM association, whereas PDLP1 is distributed along the PDom and also the PM. Furthermore, the C-terminal tail as well as the TM are necessary for PDLP5 to associate with PD (J.-Y. Lee, unpublished data), whereas the TM 
domain alone is sufficient for the PD targeting of PDLP1 [29]. It is plausible that although the lipid interaction through TM domains is an important aspect of their PD targeting, other factors may determine the specificity of PD subdomains that they each can associate with. This point may apply to other integral membrane proteins that are associated with PDom via their TM domain, including certain LRKs.

Sterol inhibition also differentially affected PD callose deposition in the outer root tissues versus in the vasculature. This effect is difficult to directly correlate with the dissipation of PDCB1 and PdBG2 from the neck regions, because although both PDCB1 and PdBG2 bind to callose and tend to concentrate at PD neck regions, they affect PD-callose levels in opposite manners $[30,31]$. The former facilitates PD callose deposition, whereas the latter inhibits it by hydrolysis. Still, sterol inhibition could prove to be a highly useful approach in determining how many different PD-proteins partition to the neck region of PDom in a given cell types and tissues.

\section{$P D$ may provide hubs for specific receptor-mediated signaling components}

Certain apoplasm-mediated, non-cell autonomous signaling components, including ARABIDOPSIS CRINKLY4 (ACR4), CLAVATA1 (CLV1), STRUBBELIG (SUB)/SCRAMBLED (SCM), and QUIRKY (QKY), are recently found to associate with PD in addition to their PM localization. CLV1, an RLK containing an leucine-rich repeat (LRR) ectodomain, plays a crucial role in stem cell maintenance, both in the shoot and root meristem. ACR4, another RLK containing a non-LRR type ectodomain, controls distal stem cell proliferation and differentiation in the root [34]. Both CLV1 and ACR4 are expressed in the distal root meristem, and function together under the control of the signaling peptide CLAVATA3/EMBRYO SURROUNDING REGION40 (CLE40), which binds to CLV1 [8]. CLV1 is mostly localized to the PM, while ACR4 is not only targeted to the PM, but also associated with PD when ectopically expressed in Nicotiana benthamiana leaf epidermal cells. SUB/SCM, an LRK containing an atypical LRR ectodomain, functions in inter-cell layer communication during tissue morphogenesis. It also regulates organ shapes and morphology of shoot and floral meristems, and the patterning of root hairs in Arabidopsis [35]. Non-cell autonomous function of SUB/SCM in mediating tissue morphogenesis requires an interaction with the membrane protein QKY [36]. QKY contains four repeats of C2-domain-a calcium-dependent phospholipid-binding module-and two TMDs embedded in the so-called phosphoribosyltransferase C-terminal region [37]. Both SUB/SCM and QKY were previously determined to localize to the PM using a conventional confocal microscopy [36,38]; however, a higher resolution confocal microscopy in conjunction with immunogold labeling revealed that both QKY and SUB/SCM are also associated with PD at both PDom and PD neck regions [9]. Although further studies are required to draw any conclusions about the significance of these proteins' PD partitioning, the possibility that apoplasmic and symplasmic signaling may not be parallel cellular processes is tantalizing.

The TM domain serves as a major molecular determinant for PD partitioning of PD-associated proteins that contain at least one TM domain. However, it is not always sufficient for the PD localization. For some PD-associated proteins, the TM domain is also important for additional functions. Replacement of the TM domain in ACR4 with that of a PM-localized but non-PD partitioning RLK abolishes the PD-association of ACR4. It will be interesting to find out if the TM domain of ACR4 is also sufficient for PD association, as shown for PDLP1. Interestingly, fluorescence resonance energy transfer (FRET) analysis measured using fluorescence lifetime 
imaging microscopy indicates that ACR4 and CLV1 form both homomeric and heteromeric complexes through their TM domain. Although these complexes can form at both the PM and $\mathrm{PD}$, they occur as higher-order complexes at PD [34]. It is yet to be discovered whether ACR4 and CLV1 are active at PD, and if so, how their activities/roles differ from those of the complexes formed at the PM. The TM domains are also necessary for the PD targeting of QKY and SUB/SCM [9], corroborating TM-dependent PD-association. However, the TM domains alone were not sufficient for PD partitioning of SUB/SCM and QKY - other domains such as the ectodomain for SUB/SCM and part of the C2-domains for QKY were also required. Thus it appears that the non-TM domains may provide molecular interfaces for stable positioning or anchoring of these proteins at PD. Molecular interactions between QKY and SUB/SCM were shown to occur at PD based on a FRET analysis, indicating that their PD partitioning might have functional significance. However, whereas the TM domains of ACR4 and CLV1 are required for both PD partitioning and complex formation, the cytosolic domain of SUB/SCM and the C2domain-containing region of QKY contribute to their intermolecular interaction. Given that QKY and SUB/SCM are not mutually dependent for their PD association [9], finding other proteins they might interact with could provide important insight into the specific mechanisms underlying their PD association.

It would also be interesting to determine whether either ACR4 or SUB/SCM is active at PD as well, and, if so, how accumulation of these RLKs at PD might impact intercellular signal trafficking. Could they function to regulate PD permeability and/or trafficking of specific non-cell autonomous proteins? Or could their accumulation at PD be necessary for the phosphorylation of a non-cell autonomous signaling protein that can be activated while passing through the cellular boundary of quiescent cells in order to control the differentiation of distal stem cells? It was previously speculated that phosphorylation of TMV MP by a host kinase at PD could help alter MP's affinity to viral RNAs, freeing viral RNAs so that they could undergo replication in the neighboring cells $[39,40]$. In the absence of phosphorylation, the MP-viral RNA complex could move farther, passing multiple cell boundaries. This subsequent cell-to-cell movement, however, would result in a futile infection because the vRNAs tightly bound to TMV MP could not be released for replication in those cells-a process that is critical for amplifying infectious viral components while spreading them throughout the host. Perhaps, there are specific non-cell autonomous proteins functioning in the distal meristem, whose movements or activities are modulated by PD-partitioned ACR4 (or SUB/SCM in its functional cellular domains).

\section{$P D$-associated proteins may partition to non-PD membranes for dual functions}

PDLP1 is known to function in restricting PD, but localizes to the PM as well as to PD. A recent study showing that PDLP1 is involved in enhancing innate immunity through stimulating callose accumulation at fungal infection sites has shed some light on the importance of the relocation of PDLP1 to the infection sites. Hyper-accumulation of callose at the pathogen penetration or wound sites in Arabidopsis is mediated by the POWDERY MILDEW RESISTANT 4, which encodes Callose Synthase 12 (CalS12) [41]—the Arabidopsis genome encodes a total of twelve callose synthases. PMR4/CalS12 is constitutively expressed and localizes to the PM, but is enzymatically inactive However, following a powdery mildew infection, PMR4-GFP relocates from the PM to the site of infection [42]. This transport is an important mechanism underlying PMR4 activation as well as the formation of a callose-filled structure called papilla in the 
invasive fungal structure called haustoria. Similarly, PDLP1-GFP signals are also found at the membranes surrounding haustoria upon a powdery mildew infection of Arabidopsis [43] (Fig. 2). The infection increases the expression of the $P D L P 1$ transcript, which in turn affects callose accumulation within the haustorial membrane encasement. However, PDLP1 partitioning to this membrane occurs transiently prior to callose biosynthesis by PMR4, suggesting that there is no direct impact of PDLP1 on PMR4 activity, per se. The papillary callose does not form in pmr4 mutants [41]. Similar to this response, haustorial callose encasement is impaired in pdlp1,2,3 triple mutants, and these mutants are more susceptible to the fungal disease compared to the wild type plants [43], indicating that papilla callose accumulation is an important immune response. pdlp1,2,3 mutants have increased PD permeability under a normal growth condition [29], but whether the PD permeability remains compromised when these mutants are infected by powdery mildew is not yet known. Along these lines, it would be interesting to see if PDLP1 participates in PD control during fungal infection and, if so, whether its PD-related function contributes to defense responses against powdery mildew.

\section{Defense signaling pathways regulate $P D$ by recruiting specific $P D$-associated proteins}

Many environmental signals and challenges alter PD dynamics [2,3]. Recent studies have just begun to unravel how various cell signaling pathways might be integrated with the regulation of PD permeability, especially during biotic stress. These suggest that PD closure is linked to basal defense via proteins that associate with or partition to PD from the PM (Fig. 2). For example, recognition of pathogenic bacteria leads to an elevation of SA concentration via ENHANCED DISEASE SUSCEPTIBILITY 1 (EDS1), which upregulates ISOCHORISMATE SYNTHASE 1 that encodes an SA-biosynthetic enzyme [12]. Cytosolic SA accumulation activates the downstream master regulator NONEXPRESSOR OF PR1, which in turn switches the transcriptional program from the normal growth and development mode to that of defense [44]. Drop And See (DANS) dye-loading assay $[13,45]$, which allows for a real-time, non-invasive, in situ measurement of cell-to-cell dye diffusion, showed that virulent pathogen infection decreases PD permeability in systemic leaves of Arabidopsis [13]. An exogenous application of SA by spraying Arabidopsis plants also results in a PD closure response within a few hours [14]. This PD closure response induced by either bacterial infection or SA treatment requires intact PDLP5 gene function as well as functional SA signaling pathway. The SA-mediated EDS1/ICS1/NPR1dependent pathway upregulates PDLP5 expression, and increased PDLP5 protein accumulation at PD restricts PD permeability via enhancing PD callose synthesis. Accumulation of PDLP5 at PD feedback-amplifies SA biosynthesis, further reinforcing PD restriction [13]. Although PDLP5 expression is inducible by the SA pathway, there is a very low, but detectable, level of expression under normal growth conditions without biotic stress. This low expression is critical for maintaining basal PD-permeability and -callose deposition, as evidenced by severe knock-down pdlp5-1 mutants that exhibit more extensive PD trafficking of both CF dye and fluorescent protein reporters and less PD callose deposition [13]. Two novel callose synthases are non-redundantly required for PDLP5-dependent basal and SA-dependent PD regulation, underscoring the important role of PD callose deposition as a physical mechanism for PD restriction, (W. Cui and J.-Y. Lee, unpublished data).

During interactions with pathogenic microbes, plant cells recognize pathogen-associated molecular patterns (PAMPs), such as bacterial flagellins or fungal cell wall fragments like chitin. 
Consistent with the critical role that PD play in innate immunity as demonstrated by PDLP5 studies, a treatment of Arabidopsis plants with fls22, a bacterial flagellin-derived peptide, induces a PD closure. However, flagellin-elicited defense responses requires FLAGELLIN SENSING 2 (FLS2), a PM-localized RLK containing an ectodomain that binds to flagellins, for the PD regulation [10]. FLS2 is a pattern-recognition receptor, perceiving bacterial pathogens at the cell surface and initiating an immune signaling cascade [46]. The fls22-induced PD closure is compromised in fls2 mutants, and FLS2-GFP is found to partition to PD in addition to its predominant PM localization, which may be critical for the protein's effect on PD permeability [10]. Similarly, the chitin-binding protein LYSIN MOTIF DOMAIN-CONTAINING GLYCOSYLPHOSPHATIDYLINOSITOL-ANCHORED PROTEIN 2 (LYM2) localizes at the PM and PD and mediate a chitin-dependent PD closure response [10]. Notably, LYM2 is required for fungal resistance in parallel to the CHITIN ELCITOR RECEPTOR KINASE 1 [47], which localizes to the PM but does not associate with PD. Based on these results, it is tempting to speculate whether localized recognition of the PAMPs by FLS2 and LYM2 (and perhaps other unidentified PAMP receptors) occurring at PD provides an effective mechanism for direct or immediate closure of PD upon microbial infection. Along these lines, it would be informative to further test whether the PD-association of PAMP receptors is required for their effects on PD permeability and plant immunity. It would also be interesting to uncover the mechanism by which FLS2 or LYM2 affects PD permeability (e.g., is the PD-callose deposition altered by FLS2 or LYM2?), and what the functional relationships might be, if any, between these receptors and the SA/PDLP5 pathway in restricting PD permeability during basal defense.

Our understanding of the relationship between PD-mediated cell-to-cell communication and biotic stress is currently limited to only the few examples discussed above. Based on these data, pathogen infection stimulates PD closure via recruiting specific proteins to PD, and lack of this response correlates with a loss of basal immunity (Fig. 2). For example, pdlp5-1 and lym2 loss-of-function mutant plants are unable to restrict PD upon infection and are more susceptible to Pseudomonas syringae pv. maculicola and Botrytis cinerea, respectively. Intriguingly, PD seem to be closed by different regulators in a manner specific to the mode of immune signaling or pathogen perception. Perhaps a particular protein or proteins are recruited as PD regulators that could interact with certain defense molecules. By adopting this mode of action, plants could not only integrate PD regulation into an array of cellular signaling pathways, but also achieve multi-level and dynamic control over cell-to-cell communication under various biotic (or abiotic) stress conditions. On the other hand, given that pathogens are thought to co-evolve with hosts, it would not be surprising to find that bacterial and fungal pathogens also produce effectors that either mimic or inhibit PD regulators to counteract plant defense responses. In this regard, some of the fungal effectors that have been shown to move cell-to-cell in rice [48,49], or yet unidentified effectors might turn out to impact the maintenance or even enhancement of PD permeability in uninfected cells prior to hyphal growth into those cells. Alternatively, pathogen effectors may interfere with defense signaling pathways, short-circuiting the PD closure response.

\section{Perspectives}

Many recently characterized PD-associated proteins include RLKs and novel receptor-like proteins that mediate non-cell autonomous signaling during cell differentiation and function in 
innate immunity, suggesting the possibility that PD form an intercellular signaling hub. The integration of immune signaling pathways with PD regulation at different levels of the signaling cascade likely indicates that PD gating is highly dynamic throughout the deployment of defense responses. The PD closure and opening during defense could also be multiphasic depending on waves of different signals, intensity, and spatial location, e.g., local infection sites versus systemic tissues. Testing these possibilities and teasing out their mechanisms will require holistic approaches combining multiple analysis tools and techniques. Given that PD are intercellular channels that are essential for sharing of nutrients and exchanging signaling molecules in plants, many more novel PD components in addition to the PM (or perhaps ER)localized receptors may likely be discovered in the coming years. Here, one of the challenges is to gain insight into their possible functions at PD and the mechanisms underlying their PD association. For this, validating PD-association of those proteins in their native cellular expression domains and fine-mapping specific membraneous subdomains within PD would be one of the essential tasks.

\section{Acknowledgements}

Special thanks go to Jae J. Yoo for his helpful comments and thorough editing of this manuscript. This work is partially supported by the grant from the National Science Foundation (IOS 0954931) and the National Center for Research Resources (5P30RR031160-03).

\section{References}

1. Sevilem I, Miyashima S, Helariutta Y: Cell-to-cell communication via plasmodesmata in vascular plants. Cell Adh Migr 2013, 7:27-32.

2. Burch-Smith TM, Zambryski PC: Plasmodesmata paradigm shift: regulation from without versus within. Annu Rev Plant Biol 2012, 63:239-260.

3. Sager R, Lee J-Y: Plasmodesmata in integrated cell signaling: new insights from development and environmental signals and stresses. J Exp Bot 2014, 65:6337-6358.

4. Wu S, Gallagher KL: Transcription factors on the move. Curr Opin Plant Biol 2012, 15:645-651.

5. Brunkard JO, Runkel AM, Zambryski PC: Plasmodesmata dynamics are coordinated by intracellular signaling pathways. Curr Opin Plant Biol 2013, 16:614-620.

6. Faulkner C, Maule A: Opportunities and successes in the search for plasmodesmal proteins. Protoplasma 2011, 248:27-38.

7. Fernandez-Calvino L, Faulkner C, Walshaw J, Saalbach G, Bayer E, Benitez-Alfonso Y, Maule A: Arabidopsis Plasmodesmal Proteome. PLoS ONE 2011, 6. e18880.

8. Stahl Y, Grabowski S, Bleckmann A, Kuhnemuth R, Weidtkamp-Peters S, Pinto KG, Kirschner GK, Schmid JB, Wink RH, Hulsewede A, et al.: Moderation of Arabidopsis root stemness by CLAVATA1 and ARABIDOPSIS CRINKLY4 receptor kinase complexes. Curr Biol 2013, 23:362-371.

9. Vaddepalli P, Herrmann A, Fulton L, Oelschner M, Hillmer S, Stratil TF, Fastner A, Hammes UZ, Ott T, Robinson DG, et al.: The C2-domain protein QUIRKY and the receptor-like kinase STRUBBELIG localize to plasmodesmata and mediate tissue morphogenesis in Arabidopsis thaliana. Development 2014, 141:4139-4148.

10. Faulkner C, Petutschnig E, Benitez-Alfonso Y, Beck M, Robatzek S, Lipka V, Maule AJ: LYM2dependent chitin perception limits molecular flux via plasmodesmata. Proc Natl Acad Sci U S A 2013, 110:9166-9170.

11. Faulkner C: Receptor-mediated signaling at plasmodesmata. Front Plant Sci 2013, 4:521. 
12. Vlot AC, Dempsey DA, Klessig DF: Salicylic Acid, a multifaceted hormone to combat disease. Annu Rev Phytopathol 2009, 47:177-206.

13. Lee JY, Wang X, Cui W, Sager R, Modla S, Czymmek K, Zybaliov B, van Wijk K, Zhang C, Lu H, et al.: A plasmodesmata-localized protein mediates crosstalk between cell-to-cell communication and innate immunity in Arabidopsis. Plant Cell 2011, 23:3353-3373.

14. Wang X, Sager R, Cui WE, Zhang C, Lu H, Lee JY: Salicylic Acid Regulates Plasmodesmata Closure during Innate Immune Responses in Arabidopsis. Plant Cell 2013, 25:23152329.

15. Schwessinger B, Ronald PC: Plant innate immunity: perception of conserved microbial signatures. Annu Rev Plant Biol 2012, 63:451-482.

16. Carella $\mathrm{P}$, Isaacs $M$, Cameron RK: Plasmodesmata-located protein overexpression negatively impacts the manifestation of systemic acquired resistance and the longdistance movement of Defective in Induced Resistance1 in Arabidopsis. Plant Biol (Stuttg) 2015, 17:395-401.

17. Ding B, Turgeon R, Parthasarathy MV: Substructure of Freeze-Substituted Plasmodesmata. Protoplasma 1992, 169:28-41.

18. Tilsner J, Amari K, Torrance L: Plasmodesmata viewed as specialised membrane adhesion sites. Protoplasma 2011, 248:39-60.

19. Robards AW: A new interpretation of plasmodesmatal ultrastructure. Planta 1968, 82:200210.

20. Robards AW: Ultrastructure of Plasmodesmata. Protoplasma 1971, 72:315-323.

21. Lucas WJ, Ding B, Vanderschoot C: Plasmodesmata and the Supracellular Nature of Plants. New Phytologist 1993, 125:435-476.

22. Radford JE, Vesk M, Overall RL: Callose deposition at plasmodesmata. Protoplasma 1998, 201:30-37.

23. Zavaliev R, Ueki S, Epel BL, Citovsky V: Biology of callose (beta-1,3-glucan) turnover at plasmodesmata. Protoplasma 2011, 248:117-130.

24. Ueki S, Citovsky V: To Gate, or Not to Gate: Regulatory Mechanisms for Intercellular Protein Transport and Virus Movement in Plants. Molecular Plant 2011, 4:782-793.

25. Ding B, Haudenshield JS, Hull RJ, Wolf S, Beachy RN, Lucas WJ: Secondary plasmodesmata are specific sites of localization of the tobacco mosaic virus movement protein in transgenic tobacco plants. Plant Cell 1992, 4:915-928.

26. Fitzgibbon J, Bell K, King E, Oparka K: Super-Resolution Imaging of Plasmodesmata Using Three-Dimensional Structured Illumination Microscopy. Plant Physiol 2010, 153:14531463.

27. Tian Q, Olsen L, Sun B, Lid SE, Brown RC, Lemmon BE, Fosnes K, Gruis DF, Opsahl-Sorteberg HG, Otegui MS, et al.: Subcellular localization and functional domain studies of DEFECTIVE KERNEL1 in maize and Arabidopsis suggest a model for aleurone cell fate specification involving CRINKLY4 and SUPERNUMERARY ALEURONE LAYER1. Plant Cell 2007, 19:3127-3145.

28. Maule AJ, Benitez-Alfonso Y, Faulkner C: Plasmodesmata - membrane tunnels with attitude. Current Opinion in Plant Biology 2011, 14:683-690.

29. Thomas CL, Bayer EM, Ritzenthaler C, Fernandez-Calvino L, Maule AJ: Specific targeting of a plasmodesmal protein affecting cell-to-cell communication. PLoS Biol 2008, 6:e7.

30. Simpson C, Thomas C, Findlay K, Bayer E, Maule AJ: An Arabidopsis GPI-anchor plasmodesmal neck protein with callose binding activity and potential to regulate cell-to-cell trafficking. Plant Cell 2009, 21:581-594.

31. Benitez-Alfonso Y, Faulkner C, Pendle A, Miyashima S, Helariutta Y, Maule A: Symplastic intercellular connectivity regulates lateral root patterning. Dev Cell 2013, 26:136-147. 
32. Zavaliev R, Levy A, Gera A, Epel BL: Subcellular Dynamics and Role of Arabidopsis beta-1,3Glucanases in Cell-to-Cell Movement of Tobamoviruses. Mol Plant Microbe Interact 2013, 26:1016-1030.

33. Grison MS, Brocard L, Fouillen L, Nicolas W, Wewer V, Dormann P, Nacir H, Benitez-Alfonso Y, Claverol S, Germain V, et al.: Specific membrane lipid composition is important for plasmodesmata function in Arabidopsis. Plant Cell 2015, 27:1228-1250.

34. Stahl Y, Wink RH, Ingram GC, Simon R: A signaling module controlling the stem cell niche in Arabidopsis root meristems. Curr Biol 2009, 19:909-914.

35. Kwak SH, Schiefelbein J: The role of the SCRAMBLED receptor-like kinase in patterning the Arabidopsis root epidermis. Developmental Biology 2006, 302:118-131.

36. Trehin C, Schrempp S, Chauvet A, Berne-Dedieu A, Thierry AM, Faure JE, Negrutiu I, Morel P: QUIRKY interacts with STRUBBELIG and PAL OF QUIRKY to regulate cell growth anisotropy during Arabidopsis gynoecium development. Development 2013, 140:48074817.

37. Fulton L, Batoux M, Vaddepalli P, Yadav RK, Busch W, Andersen SU, Jeong S, Lohmann JU, Schneitz K: DETORQUEO, QUIRKY, and ZERZAUST represent novel components involved in organ development mediated by the receptor-like kinase STRUBBELIG in Arabidopsis thaliana. PLoS Genet 2009, 5:e1000355.

38. Kwak SH, Schiefelbein J: A feedback mechanism controlling SCRAMBLED receptor accumulation and cell-type pattern in Arabidopsis. Curr Biol 2008, 18:1949-1954.

39. Waigmann E, Chen MH, Bachmaier R, Ghoshroy S, Citovsky V: Regulation of plasmodesmal transport by phosphorylation of tobacco mosaic virus cell-to-cell movement protein. EMBOJ 2000, 19:4875-4884.

40. Lee JY, Lucas WJ: Phosphorylation of viral movement proteins--regulation of cell-to-cell trafficking. Trends Microbiol 2001, 9:5-8.

41. Nishimura MT, Stein M, Hou BH, Vogel JP, Edwards H, Somerville SC: Loss of a callose synthase results in salicylic acid-dependent disease resistance. Science 2003, 301:969-972.

42. Ellinger D, Naumann M, Falter C, Zwikowics C, Jamrow T, Manisseri C, Somerville SC, Voigt CA: Elevated early callose deposition results in complete penetration resistance to powdery mildew in Arabidopsis. Plant Physiol 2013, 161:1433-1444.

43. Caillaud MC, Wirthmueller L, Sklenar J, Findlay K, Piquerez SJ, Jones AM, Robatzek S, Jones JD, Faulkner C: The plasmodesmal protein PDLP1 localises to haustoria-associated membranes during downy mildew infection and regulates callose deposition. PLoS Pathog 2014, 10:e1004496.

44. Fu ZQ, Dong X: Systemic Acquired Resistance: Turning Local Infection into Global Defense. Annu Rev Plant Biol 2013, 64:839-863.

45. Cui W, Wang X, Lee JY: Drop-ANd-See: a simple, real-time, and noninvasive technique for assaying plasmodesmal permeability. Methods Mol Biol 2015, 1217:149-156.

46. Gomez-Gomez L, Boller T: FLS2: an LRR receptor-like kinase involved in the perception of the bacterial elicitor flagellin in Arabidopsis. Mol Cell 2000, 5:1003-1011.

47. Iizasa E, Mitsutomi M, Nagano Y: Direct binding of a plant LysM receptor-like kinase, LysM RLK1/ CERK1, to chitin in vitro. J Biol Chem 2010, 285:2996-3004.

48. Khang CH, Berruyer R, Giraldo MC, Kankanala P, Park SY, Czymmek K, Kang S, Valent B: Translocation of Magnaporthe oryzae effectors into rice cells and their subsequent cell-to-cell movement. Plant Cell 2010, 22:1388-1403.

49. Giraldo MC, Dagdas YF, Gupta YK, Mentlak TA, Yi M, Martinez-Rocha AL, Saitoh H, Terauchi R, Talbot NJ, Valent B: Two distinct secretion systems facilitate tissue invasion by the rice blast fungus Magnaporthe oryzae. Nat Commun 2013, 4:1996. 
**8. Stahl Y, Grabowski S, Bleckmann A, Kuhnemuth R, Weidtkamp-Peters S, Pinto KG, Kirschner GK, Schmid JB, Wink RH, Hulsewede A, et al.: Moderation of Arabidopsis root stemness by CLAVATA1 and ARABIDOPSIS CRINKLY4 receptor kinase complexes. Curr Biol 2013, 23:362-371.

CLV1 and ACR4 form differential homomeric and heteromeric complexes at the PM and PD when transiently co-expressed in tobacco leaf cells. The TM domain of ACR4 is required for its association with PD as well as its interaction with CLV1.

*9. Vaddepalli P, Herrmann A, Fulton L, Oelschner M, Hillmer S, Stratil TF, Fastner A, Hammes UZ, Ott T, Robinson DG, et al.: The C2-domain protein QUIRKY and the receptor-like kinase STRUBBELIG localize to plasmodesmata and mediate tissue morphogenesis in Arabidopsis thaliana. Development 2014, 141:4139-4148.

SUB/ SCM and QKY localize to the PM and are also associated with PD. Immunogold labeling showed that they accumulate at both the PDom and PD neck regions. SUB/ SCM and QKY interact with each other and each requires their TM domains for targeting to PD.

**10. Faulkner C, Petutschnig E, Benitez-Alfonso Y, Beck M, Robatzek S, Lipka V, Maule AJ: LYM2dependent chitin perception limits molecular flux via plasmodesmata. Proc Natl Acad Sci U S A 2013, 110:9166-9170.

LYM2 is a GPI-anchored protein, which contains a chitin-binding ecto domain and localizes to PD. LYM2 mediates chitin-induced PD closure response and participated in basal defense against fungal pathogen. FLS2 associates with PD and mediates flagellin-induced PD closure.

*13. Lee JY, Wang X, Cui W, Sager R, Modla S, Czymmek K, Zybaliov B, van Wijk K, Zhang C, Lu H, et al.: A plasmodesmata-localized protein mediates crosstalk between cell-to-cell communication and innate immunity in Arabidopsis. Plant Cell 2011, 23:3353-3373.

PDLP5 expression is induced by SA or bacterial infection. PDLP5 accumulates at PD, decreases PD permeability via stimulating PD callose deposition, and is required for basal defense. PDLP5 is localized to the central region of PD similarly to TMV MP. When co-expressed, their fluorescent signals do not overlap in the same PD.

**14. Wang X, Sager R, Cui WE, Zhang C, Lu H, Lee JY: Salicylic Acid Regulates Plasmodesmata Closure during Innate Immune Responses in Arabidopsis. Plant Cell 2013, 25:23152329.

SA or bacterial infection induces PD closure and PD callose deposition in a PDLP5-dependent manner. SA biosynthetic or signaling mutants are compromised in PD regulation during bacterial infection. PDLP5 function requires SA- and NPR1-dependent component.

*30. Simpson C, Thomas C, Findlay K, Bayer E, Maule AJ: An Arabidopsis GPI-anchor plasmodesmal neck protein with callose binding activity and potential to regulate cell-to-cell trafficking. Plant Cell 2009, 21:581-594.

PDCB1 is a GPI-anchored protein containing X8 ecto domain and localizes to the PM and accumulates at PD neck regions. The ecto domain of PDCB1 binds to callose. Overexpression of PDCB1 increases PD callose accumulation and decreases PD permeability.

**33. Grison MS, Brocard L, Fouillen L, Nicolas W, Wewer V, Dormann P, Nacir H, Benitez-Alfonso Y, Claverol S, Germain V, et al.: Specific membrane lipid composition is important for plasmodesmata function in Arabidopsis. Plant Cell 2015, 27:1228-1250.

PD-enriched membrane fractions are obtained from suspension cultured Arabidopsis cells. Lipid composition analysis shows the PD-membrane fractions are different from the PMin that they have relatively high sterol and sphingolipids contents. Altering sterol composition dissipates PDaccumulation of GPI-anchored PD-associated proteins, PDCB1 and PdBG2. The same drug treatment has no effect on PDLP1 localization to PD, which is a type I trans-membrane protein. 
*42. Caillaud MC, Wirthmueller L, Sklenar J, Findlay K, Piquerez SJ, Jones AM, Robatzek S, Jones JD, Faulkner C: The plasmodesmal protein PDLP1 localises to haustoria-associated membranes during downy mildew infection and regulates callose deposition. PLoS Pathog 2014, 10:e1004496.

PDLP1 expression is induced by downy mildew infection. PDLP1 transiently localizes to haustoriaassociated membranes prior to papillary callose deposition. PDLP1 is important for basal immunity against mildew infection.

\section{Figure legends}

Figure 1. Structure of PD and movement of soluble molecules through PD. Subdomains of PD include: PDom, PD outer membrane; PDim (PD inner membrane); APR, appressed ER; neck region; central cavity; and cytoplasmic sleeve. Green and red balls represent soluble molecules that can move through the cytoplasmic sleeve of PD when PD are open. Increased callose accumulation from basal levels leads to a PD closure. CW, cell wall; ML, middle lamella; PM, plasma membrane.

Figure 2. Integration of immune signaling pathways with PD regulation. Recognition of different PAMPs by FLS2 or LYM2 leads to a PD closure. Whether these two receptors require callose-mediated mechanism in closing PD is not known. Elevated SA concentration in response to microbial infection induces the expression of $P D L P 5$, whose feedback amplifies SA accumulation by unknown mechanism. PDLP5 partitions to PD and activates PD-callose deposition, which results in a PD closure. PDLP1 facilitates immune responses during fungal infection through relocation to the haustorial membrane surrounding infection sites. 


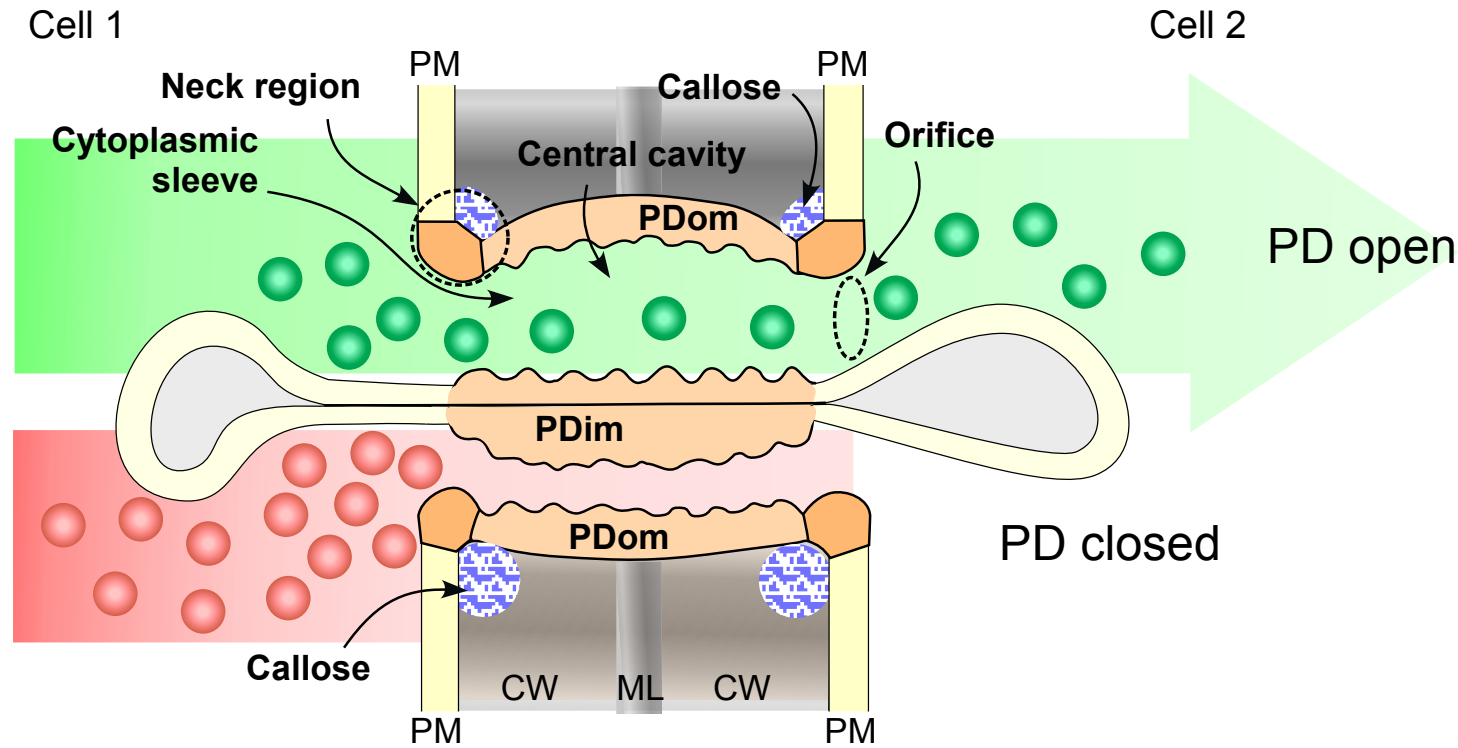




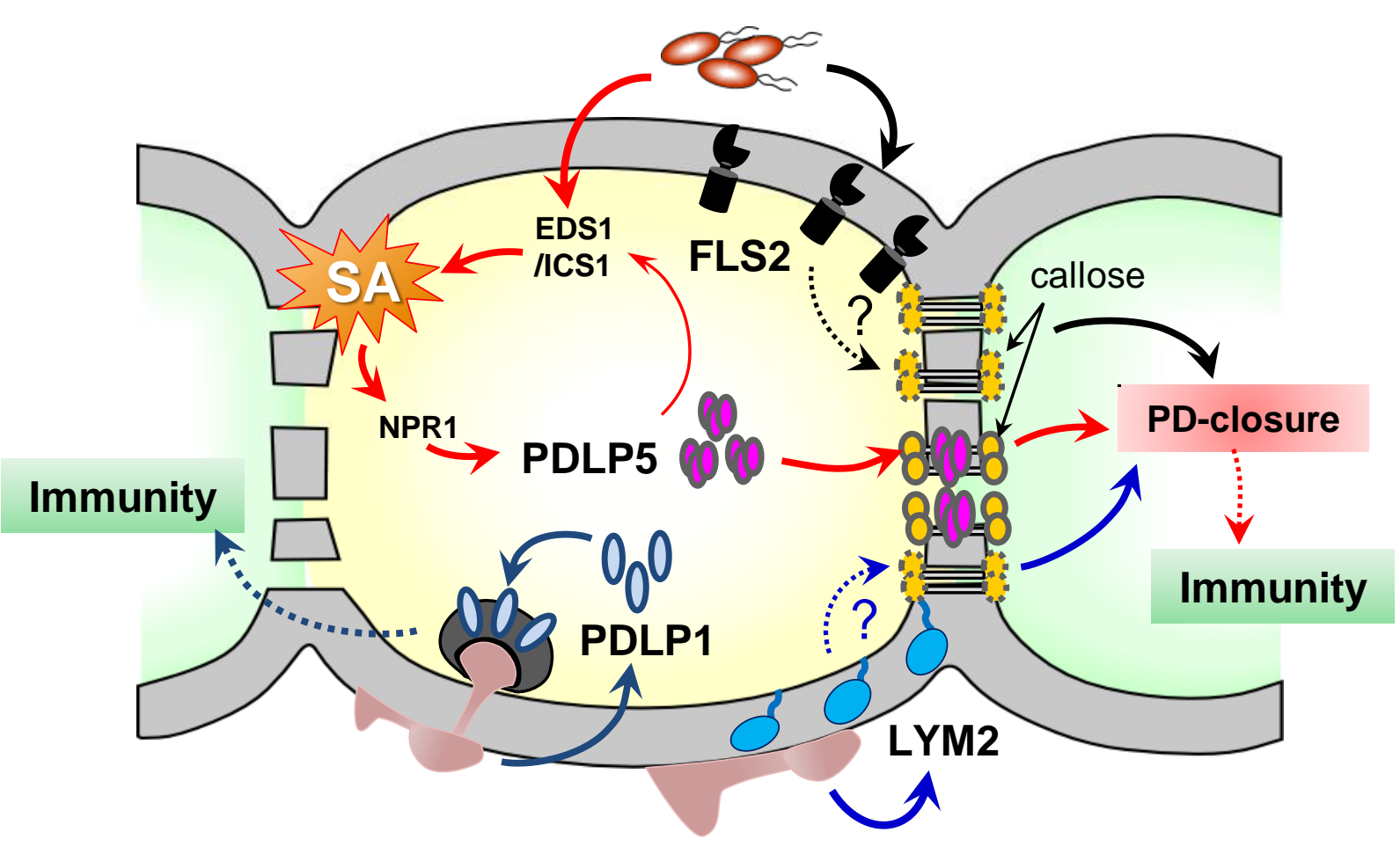

